This item was submitted to Loughborough's Research Repository by the author.

Items in Figshare are protected by copyright, with all rights reserved, unless otherwise indicated.

\title{
Solid/liquid separation by sedimentation
}

PLEASE CITE THE PUBLISHED VERSION

PUBLISHER

(C) IMechE / Professional Engineering Publishing

VERSION

VoR (Version of Record)

LICENCE

CC BY-NC-ND 4.0

REPOSITORY RECORD

Holdich, R.G., and G. Butt. 2009. "Solid/liquid Separation by Sedimentation". figshare. https://hdl.handle.net/2134/4517. 
This item was submitted to Loughborough's Institutional Repository (https://dspace.lboro.ac.uk/) by the author and is made available under the following Creative Commons Licence conditions.

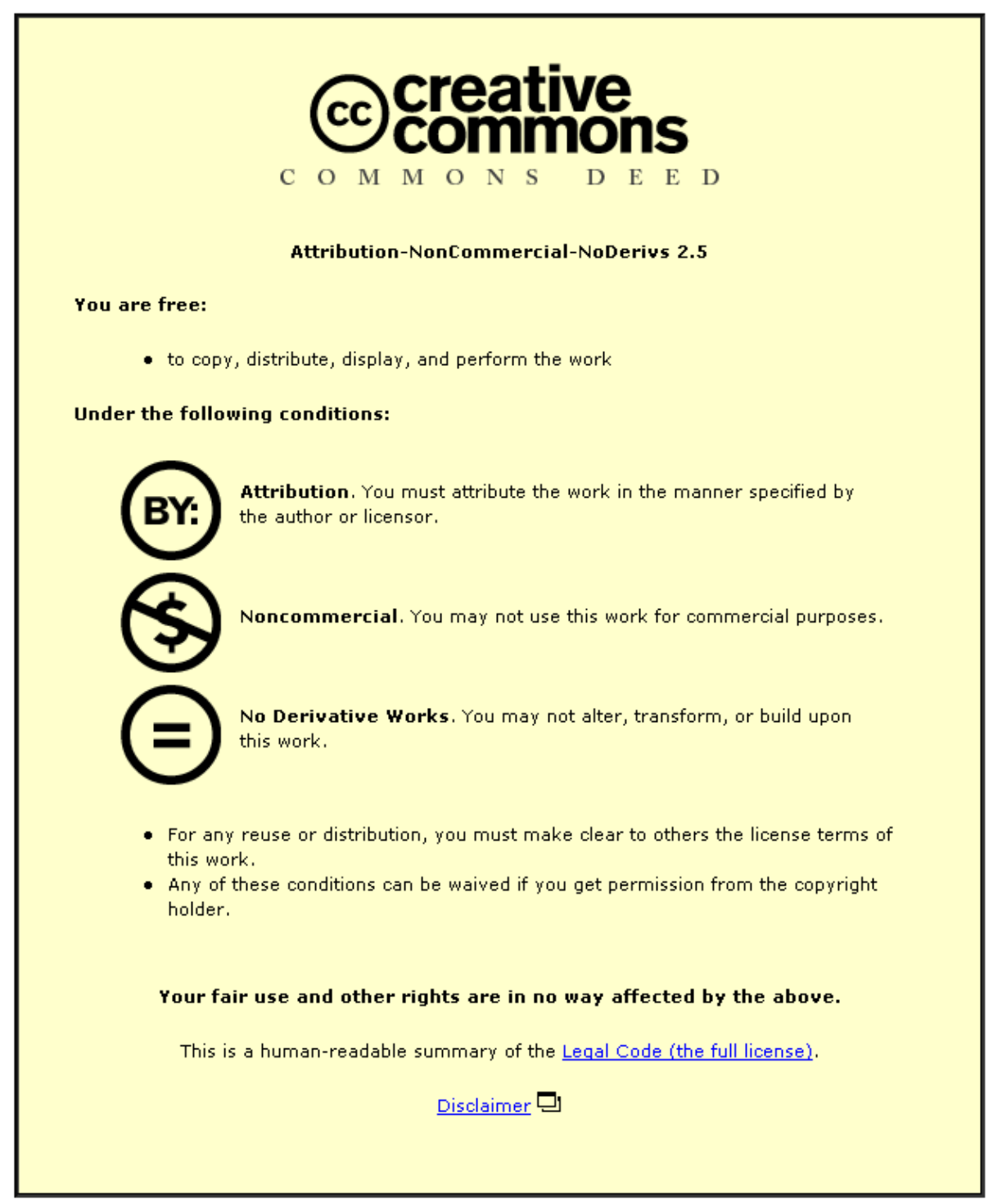

For the full text of this licence, please go to: http://creativecommons.org/licenses/by-nc-nd/2.5/ 


\title{
Solid/liquid separation by sedimentation
}

R G Holdich and G Butt

Department of Chemical Engineering, Loughborough University of Technology, Loughborough, Leicestershire

\begin{abstract}
The analysis of batch sedimentation tests performed for the purpose of continuous sedimentation vessel design is described. Conventional design techniques employ the concept of settling flux with, under certain conditions, a constitutive equation linking solid concentration and pressure in a unique and time-independent manner.

Experimental studies employing measurement of local concentration and the liquid pressure gradient during sedimentation were used to determine the limits of the conventional design techniques. A 'maximum fluidized concentration' is defined which is the value above which it is difficult to maintain stable flux conditions and is the limit of applicability of conventional design methods based on settling flux. It is shown that the suspended solids contribute towards the liquid pressure gradient and, therefore, to the buoyancy experienced by the settling solids. However, during settlement the liquid pressure gradient reverts to the hydrostatic gradient alone; hence the buoyancy effect is a function of sedimentation time. Additional considerations also suggest that a unique relation between concentration and pressure should only be used as a constituent term in a time-dependent consolidation model. Current research effort includes a suitable method of linking time-dependent consolidation theory, under conditions of extremely low applied pressure, and sedimentation flux analysis.
\end{abstract}

Keywords: sedimentation, consolidation, buoyancy, flux, thickener, pressure

\section{NOTATION}

C solid concentration by volume fraction

$C_{\max } \quad$ maximum solid concentration by volume fraction

$h \quad$ height of sediment (m)

$k \quad$ hydraulic permeability $\left(\mathrm{m}^{2}\right)$

$n \quad$ exponent on equation (8)

$P_{\mathrm{L}} \quad$ liquid pressure $(\mathrm{Pa})$

$P_{\mathrm{s}} \quad$ solids pressure $(\mathrm{Pa})$

$S \quad$ specific surface area per unit volume of particles $\left(\mathrm{m}^{2} / \mathrm{m}^{3}\right)$

$t \quad$ sedimentation time (s)

$T_{\mathrm{c}} \quad$ dimensionless consolidation time

$U$ propagation velocity of concentration characteristic $(\mathrm{m} / \mathrm{s})$

$U_{\mathrm{c}} \quad$ consolidation ratio

$v \quad$ solids settling velocity $(\mathrm{m} / \mathrm{s})$

$v_{\mathrm{f}} \quad$ fluidizing or up-flow test velocity $(\mathrm{m} / \mathrm{s})$

$v_{\mathrm{L}} \quad$ liquid velocity $(\mathrm{m} / \mathrm{s})$

$x \quad$ distance from base of column (m)

The MS was received on 22 November 1995 and was accepted for publication on 31 May 1996.

\author{
Greek \\ $\mu \quad$ liquid viscosity ( $\mathrm{Pa} \mathrm{s}$ ) \\ $\rho \quad$ liquid density $\left(\mathrm{kg} / \mathrm{m}^{3}\right)$ \\ $\rho_{\mathrm{m}} \quad$ suspension density $\left(\mathrm{kg} / \mathrm{m}^{3}\right)$ \\ $\rho_{\mathrm{s}} \quad$ solid density $\left(\mathrm{kg} / \mathrm{m}^{3}\right)$ \\ $v \quad$ consolidation behaviour index
}

\section{INTRODUCTION}

Gravity sedimentation of suspended material from liquid is an extensively practised form of mechanical separation in many industries including: water and waste-water, minerals and chemicals. The driving potential for the separation is freely available and this has led to it being the first choice in most industrial situations, provided that sedimentation time is not a constraint. It has, however, become a poor relation for research effort compared to other separations perceived to be more modern. The relative scarcity of recent research effort into sedimentation implies that the technology is mature with little more to be gained in understanding. This position is wholly unjustified; there are still some considerable challenges to understanding the sedimentation process. This paper focuses on the less well understood areas of sedimentation, describing some of the laboratory tests 
that can be used to provide a better awareness of the way in which a material sediments. The relevance of the tests to the design of commercial sedimentation vessels that often employ continuous feed and continuous discharge of clear liquid and settled sediment and are called simply 'thickeners' will also be discussed.

Firstly, the description of the sedimentation process needs to be split into two: 'clarification' at very low solid concentration and 'gravity thickening' or 'hindered settling' at higher values. The hindered settling region needs further subdivision (1), as illustrated in Fig. 1.

There are four distinct regions illustrated in Fig. 1, each region requiring a separate mathematical approach. In the clarification region particles are settling freely without hindrance from each other. The particle sedimentation rate is that of a single particle suspended in a liquid. The mathematical description of this region is well known $(\mathbf{2}, \mathbf{3})$ and so this type of sedimentation will not be further discussed here. When the particle concentration is sufficiently great the particles do not settle individually but en masse. Hence an interface between the clear liquid at the top of the vessel, or supernatant, and the settling suspension can be observed moving slowly down the sedimentation vessel. If the suspension was homogeneous initially, the rate of descent of this interface is constant when settlement is in the 'zone settling' region. The material directly below the settling interface maintains the same value as the initial concentration until shock waves of higher concentrations can reach the interface; these waves emanate from the base of the vessel. The constant settling velocity has led to the belief that the sedimentation velocity is a unique function of solid concentration: the greater the concentration the lower the velocity.

In a one-dimensional analysis of sedimentation the concentration at any height is uniform. In a homogeneous suspension the first layer at a concentration slightly greater than the original is formed at the base of the vessel; in the next instant in time the layer next

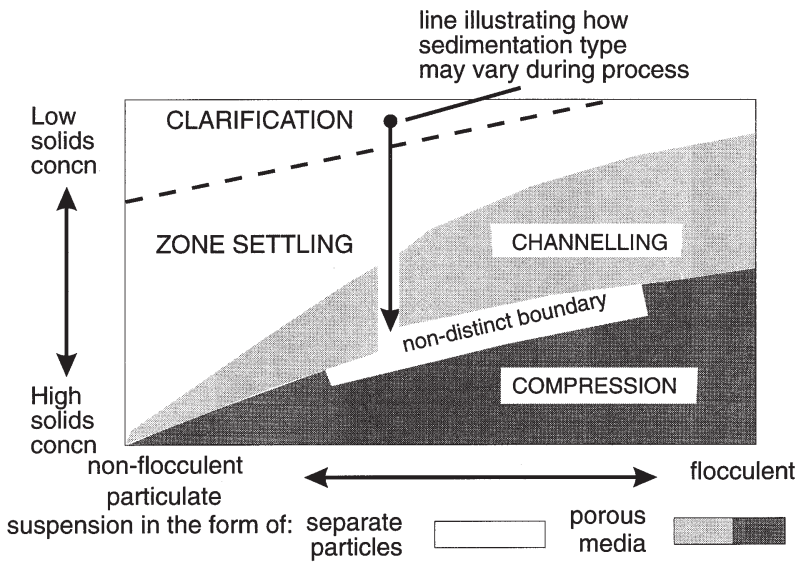

Fig. 1 Types of sedimentation behaviour according to Fitch (1) to the base has increased by a further increment in concentration. The position of the layer at the same concentration that was just above the base in the first time increment is now higher up in the vessel. In this way concentrations between the initial value and the final settled value are perceived to move upwards in the sedimentation vessel. Of course, all the solids are moving downwards; it is the position at which a certain concentration of solids exists that is moving upwards, until that position coincides with the settling interface. At this point in time the solid concentration at the interface is greater than that of the initial suspension and the interface settles more slowly. From this point onwards the concentration at the interface is continually increasing; hence the settling rate continually decreases, supporting the belief that the sedimentation rate is a unique function of solid concentration. The perceived upward motion of the intermediate concentrations is often referred to as the concentration 'characteristic', which has a fixed 'propagation' velocity from the origin. The interface settling curve and concentration characteristics for the settling of a suspension of ballotini (glass beads) at an initial solid concentration of 30 per cent by volume in water are illustrated in Fig. 2.

In compression or consolidation settling the suspension has a resistance to motion due to the mechanical strength of the compact. In this instance the suspension does not exist as discrete particles suspended in a liquid, but more like a network of solid particles or porous media with liquid in the voids. These can be displaced by the application of a suitable pressure on the solid network, the analogy with a saturated sponge sometimes being made. Between zone and compression settling a further subdivision into 'channelling settling' is visible in Fig. 1. This is a very ill defined region in which the suspension displays the properties of a connected network but may also show evidence of zones of constant concentration (at the initial value), variable concentration due to the propagation of concentration characteristics and sediment, similar to the description of zone settling. This region has not received much research

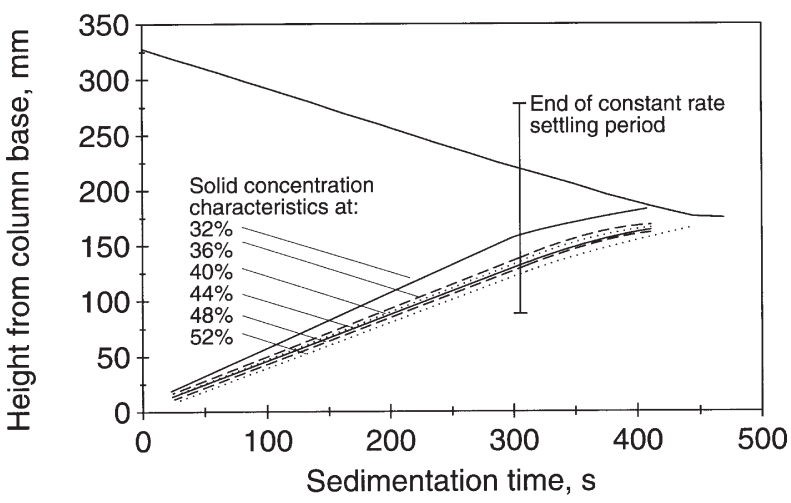

Fig. 2 Solid concentration characteristics during settling of an incompressible solid (ballotini) 
attention as the belief is that channelling augments the rate at which liquid is squeezed out of the sediment and into the supernatant. It is argued that channelling is a useful phenomenon for increasing settling rates and a design based on conditions that does not channel will, therefore, be more than adequate if channelling occurs subsequently.

\subsection{Analytical techniques}

The following mathematical analysis includes the constituent equations to describe the sedimentation in the hindered settling and compression regions described above. All the models are one-dimensional, assuming a concentration gradient in the vertical direction only. The equations are also the most simplified forms that are available. Forms employing substitutions for constituent terms or unusual coordinate systems or rendering variables dimensionless have been avoided in order to reduce the complexity of the analysis. In most instances there are two independent variables: time and height.

The laboratory tests employed to obtain design data for continuous thickeners are usually of the batch type and often employ a simple measuring cylinder. Observation of the settling interface height with time provides the settling velocity at the initial solid concentration. This can then be converted into a settling flux (solid mass per unit time and area). In continuous thickening an additional flux is due to the removal of material from the base of the vessel; this underflow withdrawal flux is usually added to the batch settling flux to give a composite value. The underflow withdrawal flux depends on how the process is operated whereas the batch settling flux is a property of the solids; hence most of the laboratory and mathematical analytical techniques investigate the batch settling velocity or flux. The following analysis refers, therefore, to the batch settling tests.

The mathematical description of batch sedimentation has been given by many research workers. The usual approach is to consider a material and a force, or momentum, balance. A material balance on the solids settling in a differential layer provides (4)

$$
v=-\frac{1}{C} \int \frac{\partial C}{\partial t} \mathrm{~d} x
$$

where $C$ is the solid concentration of the differential layer in volume fraction terms, $v$ is the solids settling velocity, $x$ is position and $t$ is settling time. A force balance (5) on the solids, neglecting inertial terms (6), provides

$$
0=C\left(\rho_{\mathrm{s}}-\rho_{\mathrm{m}}\right) g-C F_{\mathrm{d}}-\frac{\partial P_{\mathrm{s}}}{\partial x}
$$

where $C F_{\mathrm{d}}$ is the liquid drag force on the solids in the differential layer, $\partial P_{\mathrm{s}} / \partial x$ is the solids stress gradient and $\rho_{\mathrm{s}}$ and $\rho_{\mathrm{m}}$ are the densities of the solids and suspending medium respectively. There is some controversy over the density of the suspending medium: whether to use the density of the liquid or that of the mean suspension density including the solids present. This will be considered later; for the present moment the suspending medium density will be taken to refer to either option. Using Darcy's law for the dynamic liquid pressure gradient $\left(\partial P_{\mathrm{L}} / \partial x\right)$ and combining with a force balance on the liquid gives

$$
C F_{\mathrm{d}}=\frac{\partial P_{\mathrm{L}}}{\partial x}=-\frac{\mu}{k}(1-C)\left(v_{\mathrm{L}}-v\right)
$$

where $v_{\mathrm{L}}$ is the liquid velocity, $\mu$ is the viscosity and $k$ is the hydraulic permeability. Combining equations (2) and (3) gives

$$
\frac{\partial P_{\mathrm{s}}}{\partial x}=C\left(\rho_{\mathrm{s}}-\rho_{\mathrm{m}}\right) g+\frac{\mu}{k}(1-C)\left(v_{\mathrm{L}}-v\right)
$$

Now, in batch settling the combined continuity equations for the solid and liquid in a layer is

$$
C v+(1-C) v_{\mathrm{L}}=0
$$

Therefore,

$$
(1-C)\left(v_{\mathrm{L}}-v\right)=-v
$$

Hence, equation (4) can be simplified to

$$
\frac{\partial P_{\mathrm{s}}}{\partial x}=C\left(\rho_{\mathrm{s}}-\rho_{\mathrm{m}}\right) g-\frac{\mu}{k} v
$$

Equation (5) considers only three forces to be relevant: the buoyed weight of the solids in the layer, the liquid drag as a result of particle layer motion and the solids stress gradient which can transmit particle weight by point contact through a network of contacts. When fully settled only the weight and stress term are appropriate.

Equation (5) is relevant to the analysis of batch sedimentation which was qualitatively described earlier. It is also relevant to another experimental technique based on the 'up-flow' test in which liquid is passed upwards through a porous plate and the otherwise settling suspension. During the up-flow test, or fluidization, the intention is for the solid concentration to be constant throughout the vessel height; thus no solids stress gradient can exist. The only relevant forces in this instance are buoyed weight and liquid drag.

In batch sedimentation the solids material balance [equation (1)], can be rearranged as follows:

$$
v=-\frac{1}{C} \int \frac{\partial C}{\partial t} \mathrm{~d} x=-\frac{1}{C} \int_{C_{\max }}^{C} \frac{\partial x}{\partial t} \mathrm{~d} C
$$

using the chain rule. The term $\partial x / \partial t$ is the velocity at which a layer of constant concentration propagates upwards within the batch sedimentation vessel, i.e. the velocity of the solid characteristic. These are illustrated in Fig. 2. Equation (6) can be rearranged to provide an analytical expression for the characteristic velocity 
$(U=\partial x / \partial t)$ in terms of the solid settling velocity

$$
U=-\frac{\partial(C v)}{\partial C}
$$

If the solid settling velocity is a unique function of concentration, equation (7) becomes a full differential. The product of settling velocity and concentration is often called the batch settling flux and has the units of velocity. Further multiplication by solid density provides the settling flux in terms of the more usual units: mass of solids settling per unit area and time. However, solid density is independent of solid concentration and is, therefore, conventionally not used in the flux expression. The propagation velocity of the characteristic is, therefore, the differential of the batch flux plotted against the solid concentration, according to equation (7). Hence, if it is possible to obtain a unique batch settling flux curve by observing the subsidence of the suspension/supernatant liquid interface with time, then the propagation velocities of the solid characteristics can be determined. The height of a layer at any concentration between that of the initial and final values at any instant in time therefore results, i.e. the settling concentration profile at any time can be predicted. This situation represents the simplest form of sedimentation analysis that is valid for incompressible materials $\left(\partial P_{\mathrm{s}} / \partial x=0\right)$.

For compressible sediments there are several equations that relate the solid concentration to the solid pressure, such as

$$
C=C_{0} P_{\mathrm{s}}^{n}
$$

where $C_{0}$ and $n$ are empirical constants. The chain rule can be applied at any instant in time to provide

$$
\frac{\mathrm{d} C}{\mathrm{~d} x}=\frac{\mathrm{d} C}{\mathrm{~d} P_{\mathrm{s}}} \frac{\mathrm{d} P_{\mathrm{s}}}{\mathrm{d} x}
$$

Hence, with compressible materials the concentration gradient may be obtained from equation (5) and the differential form of equation (8), provided the solid settling velocity can be estimated or determined by iteration. The solid concentration profile then follows by integration.

During sedimentation the mean suspension density can be obtained from the component densities and concentrations:

$$
\rho_{\mathrm{m}}=C \rho_{\mathrm{s}}+(1-C) \rho
$$

Hence, the solid density minus the mean suspension density is as follows:

$$
\rho_{\mathrm{s}}-\rho_{\mathrm{m}}=(1-C)\left(\rho_{\mathrm{s}}-\rho\right)
$$

The term $(1-C)$ can be thought of as the buoyancy correction term on the density difference. A brief discussion on this controversial topic follows. At low solid concentration the buoyancy correction is simply that of the density difference between the solids and liquid. However, at higher values of $C$ the solids may be fully suspended in the liquid. The suspended solids will, therefore, contribute to the hydrostatic liquid pressure gradient which is the force giving rise to buoyancy. If the hydrostatic liquid pressure gradient is increased the buoyancy force will also increase. It is this principle that enables the hydrometer and dense medium separation in mineral processing to function. Hence, the buoyancy term should be the solid density minus the suspension density, or the buoyancy correction term $(1-C)$ is required for the difference between the solid and liquid densities. However, when the solids are fully settled they will no longer contribute to the liquid pressure gradient and the buoyancy correction term becomes unity. Between being fully suspended and fully settled the buoyancy correction term must have some finite, and fractional, value. In the literature most mathematical analyses of sedimentation use a buoyancy correction term of unity. The following experimental work includes some investigation into the buoyancy correction term.

One means of investigating how the suspended solids contribute to the liquid pressure gradient is to measure the hydrostatic pressure difference between two locations in a suspension. The 'excess' liquid pressure is the pressure in excess of the pure hydrostatic head, and is a measure of the amount of suspended solids present. It is derived from equation (9) and the static component of Bernoulli's equation. The excess liquid pressure $\left(\Delta P_{\mathrm{e}}\right)$ is, therefore,

$$
\Delta P_{\mathrm{e}}=\Delta h g C\left(\rho_{\mathrm{s}}-\rho\right)
$$

where $\Delta h$ is the distance between the two pressure transducers. The excess hydrostatic pressure should become less significant as solids form a network that can transmit particle weight, i.e. compression becomes important.

A means of predicting the hydraulic permeability of a suspension and how it varies with solid concentration would enable an analytical solution of equation (5). Two of the more common permeability models are due to Kozeny-Carman [for moving beds the Kozeny constant is $3.36(7)$ ]

$$
k=\frac{(1-C)^{3}}{3.36 C^{2} S^{2}}
$$

and Happel and Brenner (8)

$$
k=\frac{2-3 C^{1 / 3}+3 C^{5 / 3}-2 C^{2}}{3+2 C^{5 / 3}} \frac{3}{C S^{2}}
$$

where $S$ is the specific surface area per unit volume of the solids making up the size distributed material. The experimental procedures enabled permeability to be measured under conditions when a transient solids stress gradient may be present, and in the absence of such a stress. The procedures are described below.

Finally, techniques from the consolidation analysis of soils (9) have found some application in solid/liquid separation $(\mathbf{1 0}, \mathbf{1 1})$. There are three significant constituent equations. Firstly, the dimensionless consolidation 


$$
\begin{aligned}
& \text { ratio }\left(U_{\mathrm{c}}\right) \text { is } \\
& \qquad U_{\mathrm{c}}=\frac{h_{1}-h}{h_{1}-h_{\infty}}
\end{aligned}
$$

where $h_{1}$ is the height at which consolidation of a sediment becomes significant, $h$ is the sediment height and $h_{\infty}$ is the final settled height. The dimensionless consolidation time $\left(T_{\mathrm{c}}\right)$ is (12)

$$
T_{\mathrm{c}}=t \frac{v_{\mathrm{f}}}{h_{0}}
$$

where $v_{\mathrm{f}}$ is the fluidization velocity at the feed concentration and $h_{0}$ is the initial height of the sludge. The Terzaghi constitutive equation linking the consolidation ratio and the consolidation time is

$$
U_{\mathrm{c}}=\frac{\sqrt{ }\left(4 T_{\mathrm{c}} / \pi\right)}{\left[1+\left(4 T_{\mathrm{c}} / \pi\right)^{v}\right]^{1 / 2 v}}
$$

where $v$ is the consolidation behaviour index, which is often assumed to have a constant value of 2.85 (11).

\section{EXPERIMENTAL}

The solid materials reported in this study were ballotini, calcium carbonate in the crystal form of aragonite and talc. The true solid densities were 2923, 2590 and $2690 \mathrm{~kg} / \mathrm{m}^{3}$ and the particle diameters that have the same specific surface area per unit volume as the full size distribution were 40, 7.6 and $4.5 \mu \mathrm{m}$ respectively.

Two experimental sedimentation techniques are briefly described: batch tests in vessels $350 \mathrm{~mm}$ high and an 'upflow' test in a vessel $1 \mathrm{~m}$ high. Two sedimentation vessels were used; the internal diameters were 65 and $150 \mathrm{~mm}$. These were used to check on the effect of wall support forces; the results provided here are only those that showed insignificant wall support. The up-flow test is a liquid fluidization, in which the intention is to maintain a constant concentration throughout the vessel by pumping clean liquid upwards through a porous plate at the bottom of the vessel; the $65 \mathrm{~mm}$ diameter vessel was used in these tests. The local solid concentration during both batch sedimentation and up-flow testing was recorded by means of pairs of electrodes positioned at $10 \mathrm{~mm}$ intervals on all the vessels employed. The local electrical resistance was measured using a square wave of $2000 \mathrm{~Hz}$ frequency and converted into a solid concentration via a calibration. Scanning the columns took approximately 6 seconds and the data were stored on a computer. The experimental equipment is illustrated schematically in Fig. 3. Further details of the equipment can be found elsewhere (13).

A third experimental technique involved the use of two pressure transducers positioned within the sedimentation column, in order to investigate liquid pressure gradients. Two very sensitive diaphragm pressure transducers were used. Both faced downwards to avoid mate-

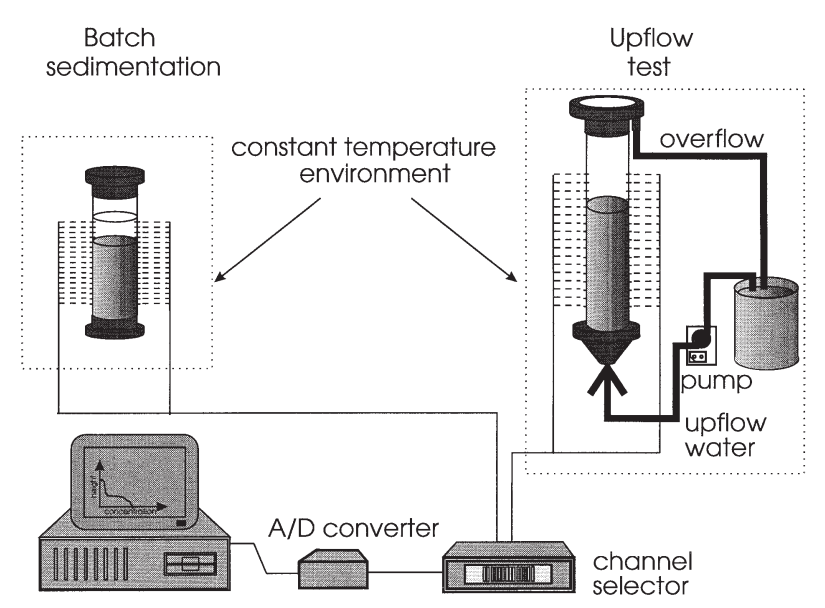

Fig. 3 Schematic diagram of sedimentation equipment

rial settling on to the face of the transducer. The bottom transducer was positioned $48 \mathrm{~mm}$ from the base of the column and the top transducer was 289 and later $252 \mathrm{~mm}$ from the base. In all the tests it was the difference between the pressures read by these two transducers that is reported. External factors such as suspension height and atmospheric pressure are not relevant as they are assumed to have a similar effect on both transducers. The pressure tests were conducted in two ways: mixing known masses of solids into suspension and measuring the pressure difference between the transducers immediately, and a similar technique followed by continuing the measurements during the ensuing sedimentation until all movement had ceased. In the latter case the concentration profile of solids in the vessel was also measured, using the technique described earlier. In all instances the pressure transducers registered the liquid pressure in the vessel which was, at first, greater than the pure hydrostatic head due to the presence of suspended solids.

\section{RESULTS AND DISCUSSION}

The batch sedimentation rig employing determination of local solid concentration was used in the production of Fig. 2. The material settled was ballotini. This material behaves in a highly incompressible manner and displays classic zone settling behaviour. A variable concentration zone, below that of the initial solid concentration zone of just over 29 per cent by volume, was measured with characteristics emanating from the origin towards the settling interface. The characteristics were close together and only six of those measured are recorded in Fig. 2. At approximately 300 seconds the settling interface slows slightly, coinciding with the first characteristic (not shown in Fig. 2) reaching the interface. Settling continued until the sediment reached a uniform concentration of 53-54 per cent by volume. At any instant in time a concentration profile, giving the local 
concentration as a function of height, can be drawn as illustrated in Fig. 4.

The incompressible nature of this material is evident from the way in which the sediment builds up. The sediment has a consistent concentration, increasing only in height at each time scan reported. The final sediment concentration is consistent with a value obtained from the random packing of a spherical material. The aragonite, however, settled in a considerably different fashion (see Fig. 5).

The aragonite sediment increased in concentration at each recorded time, eventually reaching a value of 30 per cent by volume, as well as increasing in depth. This type of behaviour might be assumed to be consistent with sedimentation in a compressible way. Finite time is required before sufficient solid mass exists in network contact to squeeze the water trapped in the voids of the sediment below. The compressible nature of this material was investigated using the equipment incorporating pressure transducers. The excess hydrostatic pressure head was calculated at a range of solid concentrations, in accordance with equation (11). The results are plotted in Fig. 6, together with the experimental measurements.

The measured excess pressures match the predicted

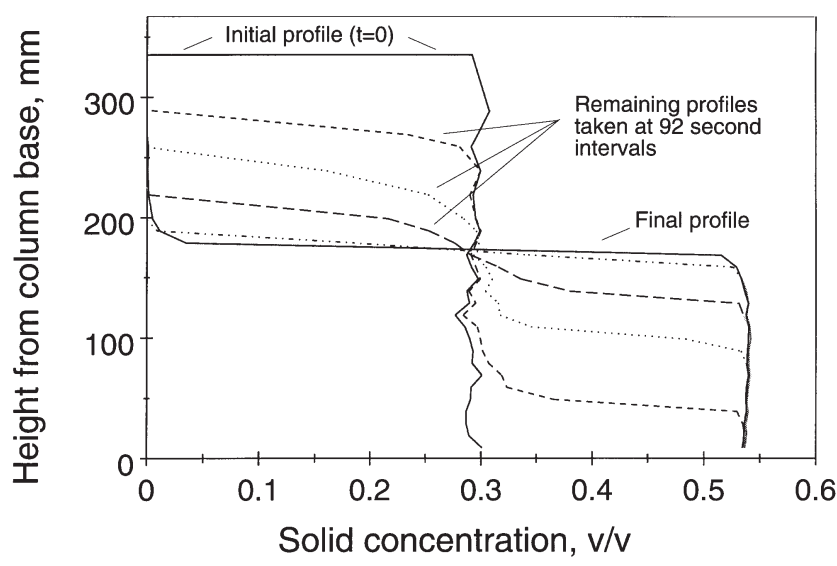

Fig. 4 Solid concentration profile during sedimentation of an incompressible solid (ballotini)

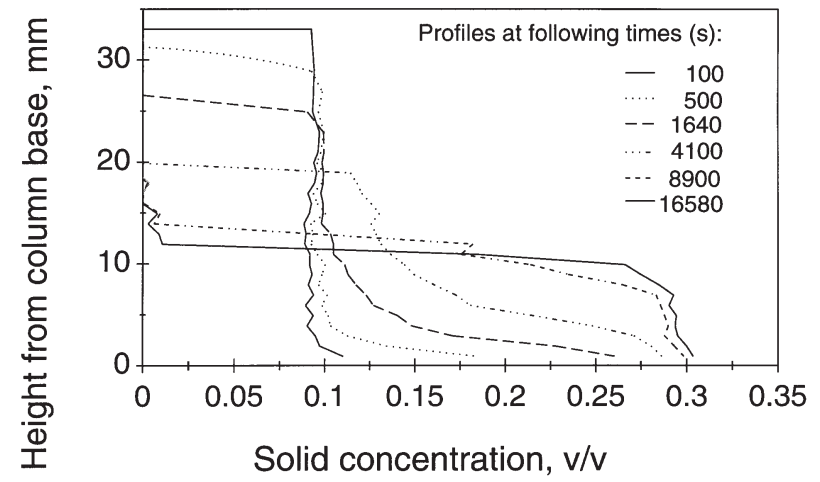

Fig. 5 Aragonite solid concentration profile during sedimentation

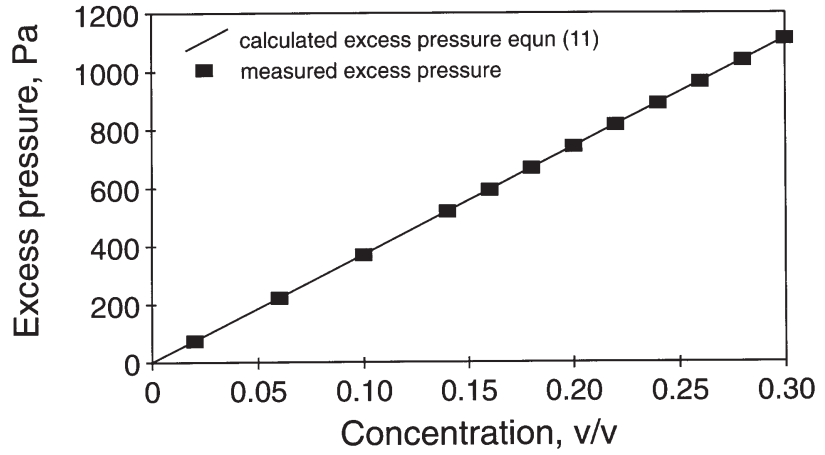

Fig. 6 Buoyancy test data for aragonite suspensions excess hydrostatic liquid pressure as a function of suspended solid concentration

values using the true solid density of aragonite in equation (11) for all concentrations. Thus all the solid weight appears to be supported by the liquid at all concentrations investigated, i.e. there is no evidence of a solid network, or compression, at concentrations up to 30 per cent by volume. This result indicates that aragonite should sediment in an incompressible manner similar to that of the ballotini. Clearly, comparison of Figs 4 and 5 shows that this is not immediately apparent. Figure 6 supports the concept that suspended solids add to the liquid pressure (hence the liquid pressure gradient and buoyancy) but does not support the concept of a unique concentration at which compressive forces become significant.

If the solids stress gradient is negligible (i.e. incompressible settling) and the concentration, densities, viscosity and settling velocity are known then equation (5) can be used to calculate the permeability. A series of batch tests at known initial concentrations were undertaken with the aragonite and the settling velocities observed. The permeability determined via equation (5) is given in Fig. 7, together with the two permeability models represented by equations (12) and (13). Also shown in Fig. 7 are the permeabilities determined from the up-flow tests. During the up-flow test the fluidization

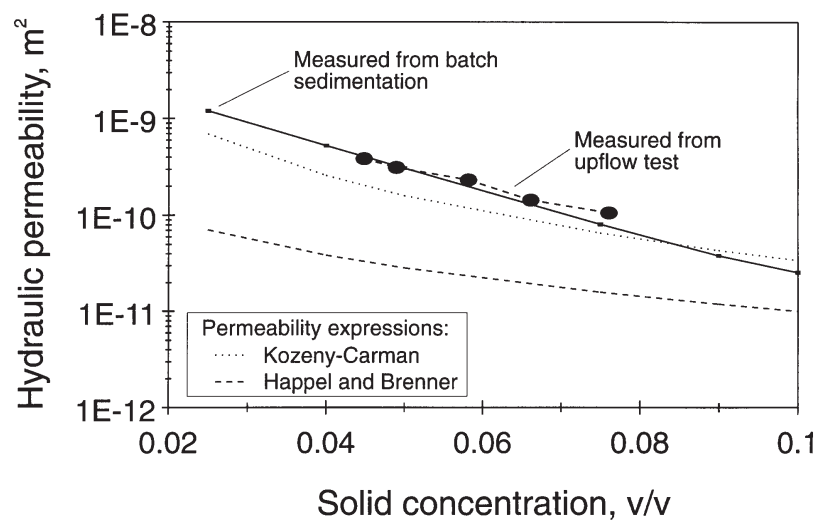

Fig. 7 Hydraulic permeability determined during aragonite sedimentations 
ensured that there were no solids concentration or stress gradients. The analysis of the up-flow test data also employs equation (5), but with a greater degree of certainty about the absence of a stress gradient.

The uniformity of the solid concentration during the up-flow test was confirmed by the local concentration data from the electrically scanned vessels. It was not possible to run the up-flow test with aragonite concentrations greater than 7.6 per cent by volume as the fluidized column collapsed; i.e. the superficial velocity to maintain concentrations of more than 7.6 per cent by volume was less than the apparent minimum fluidizing velocity. For solid concentrations greater than 5 per cent by volume, the solids settling velocities determined from the batch sedimentations were slightly less than the superficial velocity during the up-flow test. Hence, the permeabilities calculated from the up-flow tests are slightly greater in this region. This experimental data supports the argument that a solids stress does exist at concentrations in excess of 5 per cent by volume. Thus the experimental evidence from Figs 5 and 7 indicates significant compression. The evidence from Fig. 6, however, indicates that no significant solids network exists, at least at the start of the sedimentation, at concentrations up to 30 per cent by volume. Also shown in Fig. 7 are the permeability models represented by equations (12) and (13), which do not appear to fit the experimental data. However, the batch sedimentation permeabilities are close to the Kozeny-Carman model at low solid concentrations, and approaches the Happel and Brenner model at higher values not illustrated in Fig. 6.

The conventional method of estimating a continuous thickener's area is from a graphical construction on a batch flux curve. The limiting flux value comes from the intercept, on the flux axis, of a line drawn as a tangent to the batch flux curve and going through the underflow solid concentration. The product of this limiting flux and the thickener area must be equal to the product of the volumetric feed rate to the thickener and the feed volume fraction concentration, assuming that all the solids leave the thickener by the underflow. The batch flux curve for aragonite is shown in Fig. 8. The data were based on the batch sedimentations of known initial solid content and measured settling velocity. The batch flux curve can be used to infer the characteristic velocities by means of differentiation, in accordance with equation (7). Thus an illustration such as Fig. 2 can be inferred from the data provided in Fig. 8.

From the point of view of equipment design the important part of the batch flux curve is that illustrated to the right of the minimum fluidized velocity, as the tangent to the batch flux curve required to fix the limiting thickener flux is most likely to be drawn in this region. Industrial continuous thickeners have a reputation for being difficult to design and to control in operation if they are operating close to their limiting flux capacity.

E03695 (C) IMechE 1997

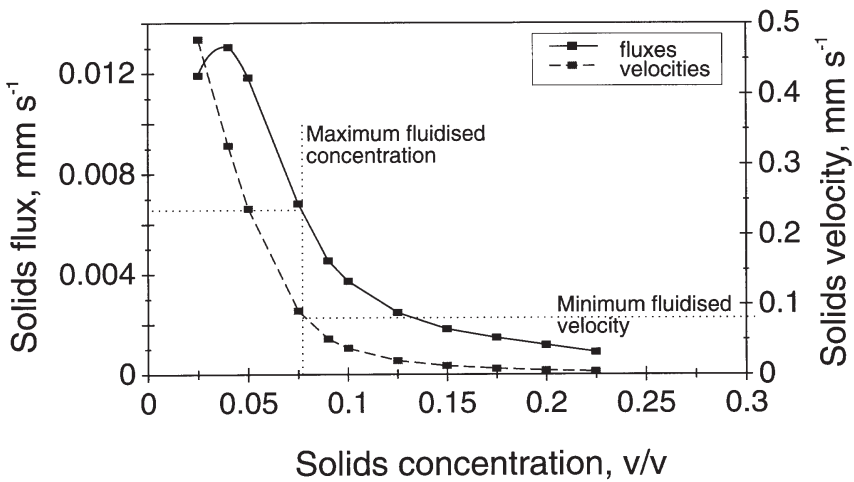

Fig. 8 Batch settling flux curve and settling velocities with concentration for aragonite

Some of the reasons behind this may be seen by reference to Fig. 8. The terms minimum and maximum 'fluidized' velocity and concentration, respectively, are used. These values are not the same as those required to achieve fluidization; for this material the minimum 'fluidizing' velocity would be the value required to fluidize solids at approximately 29 per cent solids (see Fig. 5). However, the velocity-concentration curve is very shallow until a concentration of 8 per cent solids. Thus it is extremely difficult, or impossible, to control the fluidization between 8 and 29 per cent solids; a small change in velocity considerably alters the bed concentration. This was observed in the up-flow tests. Thus the maximum 'fluidized' concentration is the value that provides stable conditions of fluidization or sedimentation. The up-flow velocity at this concentration is the minimum 'fluidized' velocity. The lack of bed stability is not immediately apparent from looking at the batch flux curve alone; the settling velocity curve is important for this assessment.

The maximum fluidized concentration is the point at which compressive forces become significant and often coincides with the point of inflection on the batch flux curve. The maximum fluidized concentration also represents the maximum point at which a continuous thickener can be designed in the conventional manner employing the flux curve. In Fig. 8 a tangent to the batch flux curve going through the maximum fluidized concentration provides an underflow concentration of 12 per cent by volume. It may be possible to operate a thickener to achieve a greater concentration than this, but the operating conditions should not be obtained from the conventional flux curve as the batch settling flux at these concentrations is time dependent.

The maximum fluidized concentration for the highly compressible suspensions formed from the mineral talc is illustrated in Fig. 9. Four up-flow liquid rates were employed: the suspended bed concentration reached 2.5 per cent by volume as the flowrate was decreased and then collapsed into a non-fluidized form. Hence for talc suspensions compressive forces and time-dependent fluxes are important from a very low solid concentration. 


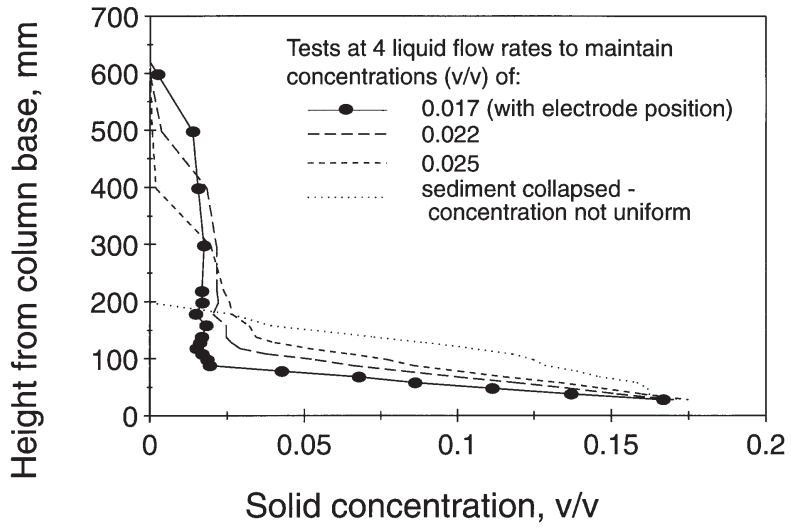

Fig. 9 Talc concentration profiles during up-flow tests

Talc suspensions were also used to further investigate the apparent lack of agreement between the expected and observed behaviour illustrated in Fig. 6. Again a series of experiments at various initial solid concentrations employing the liquid pressure measurement indicated that there was no significant variation between the excess hydrostatic head given by equation (11) and that measured, despite the apparent maximum fluidized concentration for this material being 2.5 per cent by volume. A further sedimentation test using an initial concentration of 17.7 per cent by volume, including both electrical scanning for solid concentration and liquid pressure measurement, was conducted. The sedimentation was, however, allowed to proceed to completion. At the end of the sedimentation both pressure transducers were still resident in the sediment. The result of the measured excess hydrostatic pressure and the observed interface settling height are illustrated in Fig. 10. There is no obvious relation between the interface settling curve and the pressure curve, and it should be remembered that the pressure curve is based on the liquid pressure difference between two points within the suspension. The lack of agreement between excess hydrostatic pressure and compact deformation, as provided by settling height, has been noted before (14), and will lead to serious errors

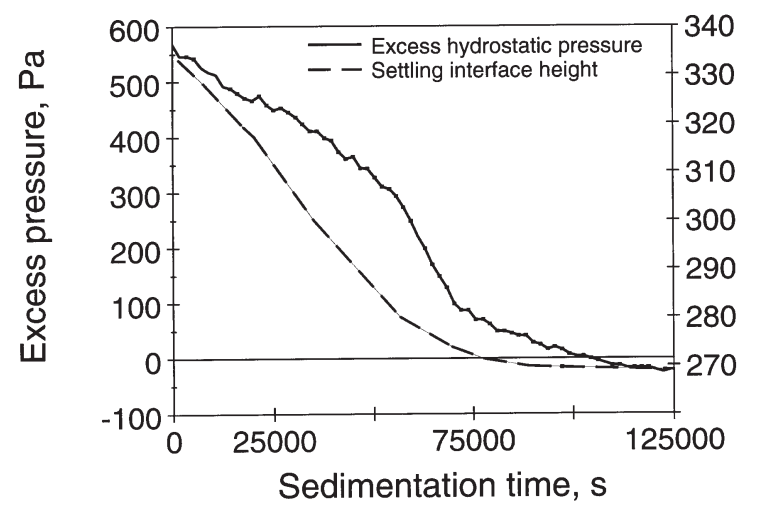

Fig. 10 Hydrostatic pressures during a talc sedimentation and interface height in attempts to infer local concentration from pressure measurement.

The initial excess hydrostatic pressure is within 5 per cent ( $30 \mathrm{~Pa}$ below) of that predicted by equation (11). After 1.5 days, at the end of the sedimentation the excess hydrostatic pressure was $30 \mathrm{~Pa}$ below the expected value of zero. The closeness with which the pressure curve approaches the expected excess hydrostatic pressure of zero, while residing in the fully settled sediment, confirms that the pressure transducers were measuring the liquid pressure alone and not the combined liquid and solid pressures. The liquid pressure variation is in agreement with the earlier discussion just prior to equation (11). The concentration profile during the sedimentation was substantially constant throughout, increasing from an initial concentration of 17.7 per cent to a final concentration of just over 22 per cent solids by volume. Thus, during the sedimentation the vessel contained concentrations between 17.7 and 22 per cent by volume but the excess hydrostatic pressure was not provided by equation (11) for these intermediate concentrations. Hence equation (11) is only valid for fully suspended materials or for compressible suspensions prior to settlement.

During the sedimentation of compressible materials both the liquid and solid pressures are dependent on the treatment history of the compressible compact; i.e. a single pressure value cannot be defined for a given solid concentration. The data illustrated in Fig. 10 can be used to illustrate this point: after 59000 seconds the concentration of the compact was approximately 20 per cent by volume and the excess liquid pressure was approximately $300 \mathrm{~Pa}$. If a suspension at an initial concentration of 20 per cent by volume was settled the excess liquid pressure would be $677 \mathrm{~Pa}$ prior to the consolidation period beginning. The variable nature of the pressures pertaining during sedimentation imply that equation (8), which states that the concentration is a unique function of the applied pressure, can only be true under certain circumstances, i.e. when there is negligible resistance to that concentration being formed. Otherwise, the concentration resulting from an applied load is both a function of pressure and time in a manner similar to conventional consolidation theory.

Conventional consolidation theory, the Terzaghi-Peck model, was provided in equations (14) to (16). The model relies essentially on establishing the concentrations at which consolidation becomes significant and at which no further consolidation with time occurs. The concentration that exists in a consolidating compact is then a function of the consolidation time and 'consolidation constants', giving rise to values between these two concentration limits.

Some research workers have combined consolidation and sedimentation models $(\mathbf{1 4}, \mathbf{1 5})$. Most of these models have been applied to suspensions of very fine particles, forming highly compressible compacts that are 
normally associated with consolidation theory. By contrast the aragonite data reported here appear to display significant sedimentation as well as consolidation behaviour under conditions of very low applied stress, i.e. overlying solid weight. Published consolidation constants for aragonite (11), obtained under compressive pressures of 2-10 bar, have been used as a first approximation to assess the significance of the consolidation of aragonite. During aragonite sedimentation, compressive pressures of 100-1000 $\mathrm{Pa}$ are typical, but consolidation times of up to 10 minutes still result from the model using the published consolidation constants. Hence, when dealing with inorganic precipitates that are in themselves incompressible a significant part of the settling process is due to the time consolidation of the compact rather than the sedimentation of the particles; i.e. the sediment concentration does not instantly jump to the equilibrium concentration that will be ultimately achieved in accordance with equation (8).

The greatest challenge in sedimentation research at present is in an appropriate method to link the classical consolidation and sedimentation flux methods in a unified model to describe the time-dependent behaviour of slightly compressible sediments. Associated with this is the time-dependent nature of solid settling velocity and batch flux. This facet affects the method of solution of equation (5) and the conventional method of designing thickeners from the batch settling flux curve.

\section{CONCLUSIONS}

The established method of describing thickener performance is by means of the batch solids flux curve, which is augmented by the underflow withdrawal flux line in continuous thickeners. It is not immediately apparent from the batch flux curve whether operating problems, such as maintaining a stable bed concentration, will be encountered in continuous thickeners. The solids settling velocity plotted against the solid concentration curve is a far better indicator of potential control problems. The up-flow test is also useful in this context as the solid concentration in the vessel is uniform during the test until bed collapse occurs. The collapse is very noticeable if the fluidizing velocity is reduced in very small increments until a massive change in bed height is given by a small change in liquid velocity. This test helps to identify the 'maximum fluidized concentration', which is the concentration above which it becomes difficult or impossible to maintain equilibrium conditions. The conventional method of calculating the area of a thickener from the batch flux curve should only be used for critical concentrations at, or below, the maximum fluidized value. At concentrations above the maximum fluidized value the batch flux is unstable and time dependent.

When the fluxes and velocities are time-dependent variables a simple constitutive relation between concentration and pressure is no longer valid. This situation is similar to that of conventional consolidation theory. The consolidation pressures are extremely small, typically of the order of $1000 \mathrm{~Pa}$, and significant time may be required in order to compress the compact to its equilibrium concentration. For the aragonite sedimentation illustrated consolidation times of 10 minutes were calculated using the Terzaghi-Peck model. The consolidation time is distinct from the sedimentation time: the former is due to the reduction in excess liquid pore pressure in the surrounding porous medium, the latter is governed only by the local conditions of concentration and, therefore, buoyed weight and permeability and hence liquid drag. However, both sedimentation and consolidation are concurrent processes and it is impossible to separate them without recourse to careful analytical techniques. Furthermore, sedimentation behaviour is linked to consolidation performance through the buoyancy correction term in the basic force balance. When fully suspended all the particle weight contributes to the liquid pressure gradient and, therefore, the suspension buoyancy. When fully settled and consolidated the particle weight acts through point contact on the surrounding particles and the buoyancy is simply due to the presence of liquid within the pores. Between these two extremes the particle weight must contribute by some fraction to the net hydrostatic liquid pore pressure and, therefore, buoyancy effect of the suspension. The excess hydrostatic liquid pore pressure during the talc sedimentations demonstrated that this pressure did not simply coincide with solids movement; i.e. there was a significant time difference between movement and consequent liquid pressure reading.

Current research into sedimentation fundamentals includes coupling consolidation theory with conventional batch sedimentation theory, with particular reference to materials that would not normally be regarded as demonstrating significant consolidation behaviour, such as inorganic and incompressible precipitates, and adapting the models to facilitate continuous thickener design or control including these insights.

\section{ACKNOWLEDGEMENT}

The authors would like to express their gratitude to the Engineering and Physical Sciences Research Council for the award of a research grant (GR/H73257) to support a project of which this work formed a part.

\section{REFERENCES}

1 Fitch, B. Sedimentation process fundamentals. Trans. Am. Inst. Min. Engng, 1962, 223, 129.

2 Stokes, G. G. On the effect of the internal friction of fluids 
on the motion of pendulums. Trans. Cam. Phil. Soc., $1851,9,8$.

3 Heywood, H. Symposium on the Interaction between Fluids and Particles, London, 1962, p. 41 (Institution of Chemical Engineers, Rugby).

4 Kynch, A. Theory of sedimentation. Trans. Faraday Soc., 1952, 48, 166-177.

5 Dixon, D. C. In Progress in Filtration and Separation (Ed. R. J. Wakeman), Vol. 1, 1979 (Elsevier, Amsterdam).

6 Wakeman, R. J. and Holdich, R. G. Theoretical and experimental modelling of solids and liquid pressures in batch sedimentation. Filtration and Separation, 1984, 21, 420-422.

7 Coulson, J. M. and Richardson, J. F. Chemical Engineering, Vol. 2, 4th edition, 1991, p. 209 (Pergamon, Oxford).

8 Happel, J. and Brenner, H. Low Reynolds Number Hydrodynamics, 1965, p. 389 (Prentice-Hall, Englewood Cliffs, New Jersey).

9 Terzaghi, K. and Peck, P. B. Soil Mechanics in Engineering Practice, 1948 (John Wiley, New York).

10 Shirato, M., Murase, T. and Iwata, M. In Progress in
Filtration and Separation (Ed. R. J. Wakeman), Vol. 4, 1986 (Elsevier, Amsterdam).

11 Wakeman, R. J., Sabri, M. N. and Tarleton, E. S. Factors affecting the formation and properties of wet compacts. Powder Technol., 1991, 65, 283-292.

12 Hoyland, G., Day, M. and Dee, A. Design procedure for sewage sludge consolidation tanks. WRc report 373-S, 1985 (WRc, Swindon).

13 Holdich, R. G. and Sinclair, I. Measurement of slurry solids content by electrical conductivity. Powder Technol., 1992, 72, 77-87.

14 Schiffman, R. L., Pane, V. and Sunara, V. Sedimentation by consolidation. In Conference on Flocculation, Sedimentation and Consolidation, Georgia, January 1985, pp. 57-121 (American Institution of Chemical Engineers, New York).

15 Shirato, M., Kato, H., Kobayashi, K. and Sakazaki, H. Analysis of settling of thick slurries due to consolidation. J. Chem. Engng Japan, 1970, 3, 98-104. 